\title{
Musique et pouvoir à l'aube de la \\ Renaissance: le metier du musicien à la \\ cour des grand Ducs Valois de Bourgogne
}

\author{
GINETTE CARTIER
}

"Lieu essentiel de la création culturelle. .., mémoire socialisée de l'imaginaire passé. .., commun aux villages et aux cours...,"1 le musicien, dans l'exercice de son métier, doit composer avec les données de sa société et de son époque. A l'aube de l'ère moderne, la civilisation occidentale, entraînée par la pression de courants nouveaux, subit une série de mutations au sein de ses structures socio-économiques et de sa vie politique, religieuse et culturelle. Ces mutations engendrent des conditionnements historiques et artistiques qui influent sur le métier du musicien, compositeur ou instrumentiste, dans ses conditions de travail et de vie matérielle, dans sa fonctionnalité et ses relations avec des employeurs issus des groupes sociaux dominants.

Les clercs savaient depuis longtemps le pouvoir des artistes et s'entendaient, par des oeuvres dont ils inspiraient les thèmes, à instruire et convaincre. A leur exemple, princes et villes surent bientôt mettre l'art au service de leur Etat ou de leur politique. ${ }^{2}$

Aussi, la problématique de cette étude consistera à comprendre dans quelle mesure le mécénat des princes, notamment celui des grands ducs Valois de Bourgogne, tend à devenir un agent de transformation du métier de musicien et de l'art musical en Europe occidentale à l'aube de la Renaissance.

Notre démarche méthodologique repose sur l'utilisation de documents d'archives $^{3}$ et de sources narratives, litteraires ${ }^{4}$ et iconographiques. ${ }^{5}$ Leur témoignage s'avère particulièrement pertinent quant à la pratique musicale du temps, l'instrumentation, les groupements d'instruments et de voix, la direction des ensembles, la fonction de la musique, les conditions de l'audition musicale et la situation sociale des musiciens.

Dans une approche pluridisciplinaire, notre cadre conceptuel s'inspire largement, en premier lieu, des données fournies depuis près d'un demi- 


\section{8 / Renaissance and Reformation}

siècle par la recherche historique et musicologique. Leur principal apport, à ce chapitre, demeure la notion de conditionnement social. Celle-ci se propose d'appréhender les interrelations dialectiques entre l'art musical et le contexte historique. Elle met en relief l'importance exercée par la demande et le rôle du public à une époque où l'art se conçoit surtout en termes pratiques et fonctionnels. Aussi, le travail du musicien et le développement de la musique restent-ils liés à leur fonction dans la société. ${ }^{6}$ Selon André Schaeffner,

Quelles que soient les innovations d'ordres divers que les musiciens de génie aient apportées, eux-mêmes n'ont pu que s'adapter à des situations données: chacun se servant du matériel instrumental de se faire entendre. ..., s'exprimant dans le cadre des formes usuelles. ..., remplissant parfois des fonctions... qui l'obligent à alimenter un répertoire spécial. ${ }^{\text {' }}$

En deuxième lieu, les sciences de la communication nous permettent d'approfondir la notion de musique, code de communication sociale comparable au langage verbal. Selon Françoise Escal,

... ces musiques utilisent des signaux, le signal étant un signe qui sert d'avertissement et déclenche une certaine conduite. Il a en effet trois caractéristiques: au niveau de l'intention, il est volontaire, contrairement à l'indice; au niveau de la structure, il a un signifié global, indivisible, équivalent à un énoncé linguistique complet; au niveau de la fonction, il est utilitaire, lié à un contexte situationnel et à l'action. Il ne dépasse pas le niveau de la communication. Il doit être perçu sans entraîner des recherches de signification. ${ }^{8}$

Par conséquent, le musicien devient un agent de transmission d'informations ponctuelles sanctionnées par la réponse concrète et immédiate des sujets sociaux auxquels le message s'adresse. Dans cette perspective, une théorie du pouvoir exigera une théorie de la localisation du bruit et de sa mise en forme dans des codes influant du même coup les formes du produit musical et le rôle du musicien. ${ }^{9}$

\section{Un milieu social propice: la Cour des ducs de Bourgogne}

La cour de Bourgogne a jeté au XVe siècle un exceptionnel éclat. Elle a visé manifestement à éclipser les autres cours princières. En ce siècle où le culte de la majesté et le souci du luxe semblent avoir l'impérieuse tyrannie d'une religion, l'Etat bourguignon prend à tâche, dirait-on, de battre tous les records. S'agit-il de racheter par la somptuosité du décor, par l'étincellement des fêtes, par la gloire des pas d'armes, par la plantureuse abondance des festins, l'absence d'une couronne royale dont on voudrait insinuer par là que l'on est digne puisqu'on dépasse en largesse ceux qui en gardent trop jalousement le monopole? On le croirait volontiers. Une mégalomanie politique parait bien se cacher derrière cette obsession constante de faire de la Maison ducale la plus 
splendide de toutes les maisons qui règnent dans la chrètienté, de se hausser au premier rang des dynasties souveraines, de faire partout vanter la richesse, la générosité, le goût de "ceux de Bourgogne."10

Voilà qui situe et résume le souvenir laissé par la Maison de Bourgogne à l'apogée de sa puissance au XVe siècle, plus précisément sous le règne de Philippe le Bon et de Charles le Téméraire. Cette puissance s'explique d'abord en raison de l'accroissement progressif des possessions ducales, amorcé sous le premier duc, Philippe le Hardi. Outre l'apanage de Bourgogne, ces possessions incluent les territoires les plus riches de l'Occident, en l'occurence les Pays-Bas septentrionaux, reconnus pour leur excellente situation géographique à l'embouchure du Rhin, de la Meuse et de l'Escaut, et pour leur population nombreuse et industrieuse. La réunion politique permet l'établissement d'une administration régulière, relativement perfectionnée, et favorise l'essor des communications. A cela s'ajoute une paix relative à l'intérieur du pays. La politique ducale laisse mourir aux frontières les remous des guerres franco-anglaises, épargant ainsi aux sujets les épreuves subies par le reste de la mouvance française et favorisant, par conséquent, le développement économique. Ces bases politiques et socio-économiques permettent l'ascension d'une classe moyenne bourgeoise qui, au sein de la société urbaine, domine les formes nouvelles d'appropriation du temps. ${ }^{11}$ La richesse, la compétence et le sens de l'organisation de cette bourgeoisie d'affaires permettent le faste déployé par les ducs de Bourgogne. Toutefois, si elles le soutiennent, elles n'en expliquent pas les motivations profondes. Aussi faut-il les chercher ailleurs, à un niveau plus subtil, dans l'esprit de l'époque. L'historien Johan Huizinga explique:

... toute expérience avait encore ce degré d'immédiat et d'absolu qu'ont le plaisir et la peine dans l'esprit d'un enfant. Chaque acte, chaque événement était entouré de formes fixes et expressives, élevé à la dignité d'un rituel... les événement de moindre importance, eux aussi. . . étaient accompagnés d'un millier de bénédictions, de cérémonies et de formules.

On jouissait plus avidement de la richesse et des honneurs, car ceux-ci contrastaient plus encore que de nos jours avec la misère environnante. . Et toutes les choses de la vie jouissaient d'une publicité, ou pénible ou orgueilleuse. . . Chaque état, chaque cadre, chaque profession était reconnaissable à l'habit ..., tout s'annonçait clairement par des cortèges, des cris, des lamentations et de la musique. . .

Les formes symboliques et les contrastes perpétuels avec lesquels toute chose se présentait à l'esprit donnaient à la vie quotidienne une émotivité qui se manifestait par ces alternatives de désespoir ou de joie délirante, de cruauté ou de profonde tendresse, entre lesquelles oscillait la vie au moyen âge. ${ }^{12}$ 
La Cour constitue le terrain le plus propice au déploiement ostentatoire de cet esthétisme. Le pouvoir de suggestion des signes extérieurs renforce la fidélité au prince et assure son prestige au-delà même de ses frontières. Selon le chroniqueur Georges Chastellain, après la gloire guerrière, le faste de la Cour constitue la première chose sur laquelle on porte les yeux. ${ }^{13} \mathrm{Le}$ renforcement du pouvoir de suggestion que traduit ostensiblement la magnificence dont on entoure la personne du prince, s'apparente, dans une certaine mesure, à un phénomène de caractère hypnotique puisqu'il retient et attire l'attention des sujets par une sorte d'attraction irrésistible provoquée par des moyens artificiels de concentration sensitive. A propos de Philippe le Bon, l'historien Joseph Calmette écrivait:

Le duc aime le faste, les bijoux, les beaux chevaux et sait s'en parer, ses entrées solennelles dans les villes éblouissent les foules. Il surpasse dans l'organisation des fêtes, joutes, tournois, banquets, tout ce qui a été fait avant lui. II stupéfie ses hôtes par l'étalage de ses joyaux, de ses tapisseries, de sa vaisselle, de ses coffres pleins d'or. ${ }^{14}$

L'affirmation du pouvoir du prince et de son entourage se manifeste d'abord au plan de la réalité sonore. La considération dont ils jouissent se mesure proportionnellement à leur capacité de provoquer du bruit d'abord quantitativement par les tintements et les cliquetis. Ainsi, une mode extrêmement répandue au XVe siècle consiste à charger de campanes et de clochettes non seulement les housses des chevaux des nobles personnages mais aussi leurs propres vêtements:

La Hire porte un manteau rouge tout chargé de clochettes d'argent, semblables à celles que portent les vaches. A une entrée, en 1465, le capitaine Salazar est accompagné de vingt hommes d'armes dont les chevaux ont le caparaçon garni de grosses clochettes d'argent. . . Charles le Téméraire apparaît dans un tournoi vêtu d'un habit de fête cousu de florins; des gentilshommes anglais ont des vêtements cousus de nobles sonnants. Aux fêtes nuptiales du comte de Genève à Chambéry en 1434, un groupe de seigneurs et de dames exécutent une danse; ils sont vêtus de blanc relevé "d'or clinquant": les hommes ont de larges ceintures garnies de sonnettes. ${ }^{15}$

Même surcharge au plan visuel: les orfèvres et les tailleurs couvrent exagérément les vêtements d'orfèvrerie et de pierreries. ${ }^{16}$ Cette fois, au plan qualitatif du bruit, l'usage fonctionnel de la musique contribue à l'acceptation de l'art musical comme une activité nécessaire de l'homme civilisé incarné par le prince.

\section{La localisation du bruit: l'instrumentation musicale}

L'education et les goûts musicaux des princes jettent quelques lumières sur la classification des instruments de musique à la cour. D'un part, les 
"hauts" instruments se caractérisent par des valeur de virilité masculine évoquant les guerres et les manifestations chevaleresques visant à satisfaire l'orgueil des hommes. D'autre part, les "bas" instruments, plus raffinés, voire dénués de toute connotation virile, s'associent aux femmes et aux enfants. ${ }^{17}$

Si la cloche évoque la communauté chrétienne, un autre son centripète marque le pouvoir, le commandement, l'autorité laïque et militaire: la trompe. En effet, par sa forme acoustique déterminée par une courbe logarythmique projettant le son dans l'infini sans jamais revenir sur luimême, la trompe et les instruments de musique de sa famille impliquent une projection de l'autorité vers l'extérieur. ${ }^{18} \mathrm{Par}$ la virilité voire l'agressivité de son timbre, elle exprime les bienfaits de la victoire, la félicité du devoir accompli et la gloire du pouvoir. La trompe demeure l'instrument de l'éclat, de la puissance et de la domination. A cet élément acoustique s'ajoute un élément rythmique: le tambour. Au méme titre que la trompe qui ne reste, à tout fin utile, qu' un prolongement sonore de la voix humaine, le tambour ne rythme que plus fortement le pas puisque son battement ne s'éloigne pas de la pulsation cardiaque normale. S'inspirant des chroniques de l'époque, J. Huizinga montre le rôle significatif de ces deux types d'instruments:

Pendant la nuit qui précéda Azincourt, les deux armées, l'une en face de l'autre dans l'obscurité, s'excitaient au combat à son de trompettes, et les Français se plaignirent de n'en avoir pas eu assez "pour eulx resjouyr", ce qui fut cause que leur courage fut moins soutenu. . .

Vers la fin du XVe siècle, les lansquenets introduisirent l'usage du tambour, d'origine orientale. Avec son effet hypnotique et inharmonieux, le tambour symbolise la transition entre l'époque de la chevalerie et celle de l'art militaire moderne; il est un élément dans la mécanisation de la guerre. ${ }^{19}$

Ainsi, les trompettes et le groupe des "hauts" instruments avec les percussions occupent une fonction de glorification, de symbolisation acoustique et rythmique du pouvoir. Par contre, les "bas" instruments, à sonorité plus douce, tels la harpe, le luth et la vièle, remplissent une fonction essentiellement axée vers le simple divertissement.

\section{L'organisation du bruit: la production musicale}

$\mathrm{Au}$-delà de l'instrumentation, la musique reste le meilleur document parmanent capable d'évoquer les sons du passé et, donc, de rendre compte du paysage sonore.

La fonction et la destination sociale pratique déterminent la forme du produit musical. ${ }^{20}$ Les mentalités et les consciences individuelles et collectives utilisent, par le canal de la musique, un vaste appareil symbolique. 
"Exposer des idées, fortifier des sentiments, susciter des passions, voilà ce que, aux XIVe et XVe siècles, on attend d'un art qui n'est jamais gratuit." ${ }^{21}$ A la cour bourguignonne, elle doit satisfaire des besoins d'édification et de divertissement, ajoutant splendeur et pompe aux cérémonies et autres solennités. Il ne s'agit donc pas d'une musique de concert au sens moderne du terme:

\begin{abstract}
Religieuse ou profane la musique se mêle aux activités journalières les plus diverses de la cour, messe chantée chaque jour devant le prince et les grands, cérémonies et repas mêlés de concerts; à la guerre, en voyage, les musiciens suivent le prince. Tout est occasion de chants, de sonneries et de danses. Comme le duc de Bourgogne, les ducs d'Orléans, de Savoie, d'Anjou, de Bourbon entretiennent tous des chanteurs d'église et des joueurs d'instruments; les princes étrangers, qu'ils soient de Hollande, de Bavière, d'Italie, d'Aragon, de Portugal ne sont pas moins soucieux du plus bel ornement de leur cour. Ils savent la considération que leur vaudra un riche personnel musical. ${ }^{22}$
\end{abstract}

La composition, l'instrumentation et l'exécution musicale exerceront une influence déterminante sur le processus de différenciation, d'une part, entre les types de musiciens à savoir les compositeurs au rang social supérieur et les simples instrumentistes occupant une position sociale inférieure, et d'autre part, sur le processus de hiérarchisation à l'intérieur même de cette dernière catégorie à savoir les ménestrels de hauts et de bas instruments. Mais d'abord, le déploiement du faste de la Cour, le renforcement du pouvoir de suggestion des signes extérieurs de la puissance du prince, l'environnement acoustique dont il s'entoure et les formes musicales d'édification et de divertissement qu'il exige, amèneront une sédentarisation croissante du musicien se traduisant par un contrat de domesticité entre lui et le pouvoir, puisque "Qui dit 'fonction,' dit nécessité; qui dit 'fonction,' dit encore 'subordination.' "'23

\title{
Les compositeurs bourguignons: les chapelains de la cour
}

Dès le XIIIe siècle, le morcellement vertical des métiers, issu de l'établissement d'une dernière frontière de l'estime et du mépris entre le travail manuel et le travail intellectuel, favorise la formation d'une nouvelle "aristocratie" s'appuyant sur les formes écrites et visuelles de transmission du savoir. ${ }^{24} \mathrm{Ce}$ nouveau patriciat se compose de clercs et d'universitaires parmi lesquels se trouvent les théoriciens de la musique que la tradition antique considère comme les "vrais" musiciens. Ce mot, tel un titre honorifique, semble réservé exclusivement aux initiés, en opposition aux praticiens de la musique, non considérés comme musiciens puisque désignés par un terme formé du nom de l'instrument leur servant à produire des sons, par exemple vielleurs, harpeurs, trompettes. Ainsi s'oppose aux "vrais" musiciens un réservoir énorme d'exécutants auxquels les premiers 
ne reconnaissent que peu de culture. Le mode d'apprentissage élargit la dichotomie entre ces derniers et les instrumentistes. Les théoriciens reçoivent une éducation musicale théorique dispensée dans les universités, et les praticiens un enseignement pratique reposant sur l'audition et la manipulation des instruments. Cependant, à la fin du Moyen Age, une demande sociale grandissante de la part des groupes sociaux dominants exige une nouvelle orientation pédagogique axée vers la satisfaction des besoins que dispenseront bientôt les maîtrises des cathédrales et les chapelles princières. ${ }^{25}$ Le musicien parfait reste celui qui sait mais le savoir auquel il se réfère correspond à la science des règles de la musique et de leur application que constitue l'art du contrepoint, et non plus la science mathématique des proportions et de la connaissance de l'univers. Par conséquent, le profil du "vrai" musicien se transforme: l'ère du théoricien rompu aux disciplines du Quadrivium se termine alors que s'ouvre celle du compositeur soumis aux impératifs de la commande sociale. A ce chapitre, la coutume oppose les hommes de cour tels Gilles Binchois et Antoine Busnois aux hommes d'église tels Guillaume Dufay et Jean Ockeghem. ${ }^{26}$ Les hommes de cour déploient leurs activités au sein d'un milieu où la recherche du plaisir domine tout autre quête. Aussi la composition de pièces profanes prendra-t-elle le pas sur la musique sacrée. Toutefois, tout au long de leur règne, les ducs de Bourgogne chercheront à accroître la splendeur de leur musique religieuse. Sous Philippe le Hardi, la chapelle ducale fait l'objet de soins constants. Nuit et jour, un nombre considérable de musiciens célèbrent le service divin en son hôtel. Les grandes orgues du duc à Dijon et à Gonflans-lès-Paris ajoutent à la solennité des cérémonies. Son petit-fils, Philippe le Bon, fonde en 1422 et en 1431 deux grandmesses quotidiennes à la Sainte-Chapelle de Dijon. Il y établit une maîtrise d'enfants de choeur ainsi qu'à l'église Saint-Pierre de Lille. En 1424, il soutient la construction des orgues les plus grandioses de l'époque, celles de Notre-Dame d'Amiens. Chacun de ses châteaux possède un orgue approprié aux dimensions de la chapelle. De même, ne considère-t-on pas qu'aucune chapelle privée n'égale celle du Grand Duc d'Occident?

Les ducs de Bourgogne réuniront les meilleurs compositeurs, chantres et organistes de leur époque. A l'exemple du personnel de la cour et selon les ordonnances ducales, le personnel ecclésiastique doit escorter le duc dans ses déplacements, en temps de guerre comme en temps de paix, afin d'assurer le maintien des services religieux quotidiens. ${ }^{27}$ De même, certains comptes soulignent leur participation aux opérations militaires. Entre deux combats, les membres de la chapelle s'emploient à distraire le duc. $^{28}$

D'une part, leur musique doit souligner les grands moments de la vie chrétienne. D'abord, la naissance d'un héritier reste un événement que l'on désire marquer d'une empreinte toute solennelle. Pour celle d'Antoine de 


\section{4 / Renaissance and Reformation}

Bourgogne, premier enfant de Philippe le Bon et d'Isabelle de Portugal, né en 1430, le chapelain Gilles Binchois compose le motet Nove cantum melodie, lequel contient d'ailleurs tous les noms des membres de la chapelle ducale du temps. ${ }^{29}$ Lors des cérémonies nuptiales, le rôle des musiciens de la chapelle se résume aux célébrations liturgiques et dans une participation occasionnelle des chantres aux entremets des banquets. Enfin, si le service funèbre du prince exige à la fois le port de vêtements de deuil et le silence des instruments profane, la musique sacrée, véritable décor sonore de circonstance, suscite une forte impression, amplifiant du même coup le sentiment de douleur saisissant les spectateurs de cette sombre représentation esthétique.

D'autre part, la musique des compositeurs bourguignons reste liée aux manifestations d'une certaine psychologie collective. En premier lieu, à la façon d'une mode, l'affirmation des valeurs socio-politiques se traduit, pour tout membre de la haute aristocratie, par la création d'un ordre de chevalerie. Aussi, Philippe le Bon fonde-t-il le sien, l'ordre de la Toison d'or, en 1430, le jour de son mariage avec Isabelle de Portugal. A la fin du Moyen Age, l'ordre de chevalerie ne reste plus qu'une reproduction sécularisée des ordres religieux issus des Croisades. Si le caractère spirituel moral s'efface devant le goût du prestige et du faste, le rituel chevaleresque demeure assez riche pour donner à ces fêtes un style empreint de vénération et de solennité. Par conséquent, un produit musical de circonstance imprimera à ces manifestations un caractère sacré traduisant au plan sonore la signification de l'idéal de chevalerie. D'ailleurs, il existait un livre de chant "où sont aucunes messes pour servir à la messe de l'Ordre de la Toison d'or," pour lequel le compositeur reçut rémunération. ${ }^{30}$ En deuxième lieu, compromis entre les anciennes traditions de la culture populaire et les croyances chrétiennes, les fêtes liturgiques contribuent à renforcer les valeurs socio-religieuses. A la cour de Bourgogne, les cérémonies liturgiques requièrent le concours des musiciens de la chapelle. Souvent, pour l'occasion, le duc invite prélats et chapelains à sa table. Notamment, le cycle pascal, avec les fêtes de Pâques, de l'Ascension, de la Pentecôte et de la Fête-Dieu, reste le prétexte à de grandes heures liturgiques. Ces fêtes solennelles s'apparentent dans leur forme à des spectacles mettant en scène un très grand nombre de personnages, clercs et diacres, parés pour la circonstance de costumes colorés, et dont la musique sacrée, par la résonnance populaire des chants d'église, permet une plus étroite et spectaculaire participation des foules. Outre leur présence aux services religieux, les chantres participent aux représentations de mystères, véritables outils pédagogiques permettant d'améliorer la compréhension des fidèles en matière de foi religieuse. ${ }^{31}$ En troisième lieu, les fêtes burlesques, parfois licencieuses, constituent l'un des aspects les plus caractéristiques de l'époque. Essentiellement ecclésiastique, cette fête des Fous, des Sots 
ou des Innocents du 28 décembre demeure un simple moment liturgique, une cérémonie d'illustration religieuse dont le but consiste à mettre en scène un monde hors des normes habituelles, le renversement des hiérarchies, la revanche des ordres mineurs et des diacres sur l'évêque et les chanoines. Les simples clercs, maîtres de l'Eglise, célèbrent les offices épiscopaux à leur manière et procèdent à l'élection d'un évêque des Fous. Même Philippe le Bon encourage, subventionne et protège ces fêtes de la contestation. ${ }^{32}$

$\mathrm{Au}$ plan extra-musical, la formation académique des membres de la chapelle et leur appartenance à l'ordre ecclésial leur permettent d'occuper des charges politiques, ecclésiastiques, domestiques et diplomatiques. D'abord, la direction de l'Office de la Chapelle confère à son titulaire, en l'occurence le premier chapelain, la dignité de membre du conseil ducal. De même, les autres chapelains exercent la fonction d'aumôniers auprès du duc et de son fils, le comte de Charolais. Soulignons que les chapelains constituent autant de grands personnages que les prébendes conférées par le duc de Bourgogne anobliront et enrichiront. Les nominations de chanoines, de prévôts et de doyens, sous le couvert de récompenses honorifiques, s'inscrivent dans le cadre plus large de l'appui du clergé au pouvoir ducal et, par conséquent, de son rôle dans l'organisation politique et administrative. La mentalité particulariste et "républicaine" des Etats du Nord pèse comme une menace sur les clercs et leurs biens.

Seul le pouvoir ducal leur offre une sécurité relative. Aussi, le clergé embrasse-t-il sans hésiter le parti du duc et lui témoignera, d'une part, un appui d'ordre spirituel précieux tels que les interdits jetés sur les communes révoltées, les indulgences manifestées à l'égard de la vie privée du duc et de son entourage et, d'autre part, un appui d'ordre politique qui permettra au duc d'étendre son autorité sur tous les Pays-Bas par le biais du contrôle des nominations épiscopales. De plus, ce contrôle s'avèrera une méthode de maintien de zones d'influences contre la France et d'imposition d'un protectorat bourguignon du côté de l'empire. Aussi, revêt-il des aspects économiques importants. En fait, le duc dispose lui-même du soin d'établir le nombre de dignités ecclésiastiques, se servant d'elles pour rémunérer un personnel fortement multiplié par le développement de la cour et des institutions centrales bourguignonnes. ${ }^{33}$

En dernier lieu, les clercs de la chapelle, plus modestes, occupent leur fonction de valets de chambre. Pour eux, cette charge reste avant tout une occupation temporaire. Leur formation académique leur permet d'aspirer et d'accéder au poste de secrétaires et, plus tard, de gravir les échelons de la hiérarchie cléricale et politique. D'autres, cependant, indifférents aux positions élevées, peuvent choisir un tout autre état de vie tel que le mariage, évidemment permis pour les clercs de chapelle qui ne désirent pas la prêtrise. 


\section{6 / Renaissance and Reformation}

Ces véritables fonctionnaires cléricaux se voient parfois confier certaines missions diplomatiques et secrètes. Leur éducation les prédispose à exercer ce type d'activité au profit de l'autorité religieuse ou profane à laquelle ils se soumettent. Il ne s'agit pas d'une profession ou d'une carrière mais seulement d'un travail occasionnel, d'une mission temporaire. D'autres, soucieux d'enrichir la bibliothèque ducale, agissent à titre d'intermédiaires négociant les acquisitions et les réparations des livres. Certains composent et notent leurs messes et "passions," écrivent des oeuvres littéraires, didactiques et des nouvelles d'une moralité douteuse.

Au chapitre de l'administration interne, la chapelle ducale possède un gouvernement autonome dirigé par le premier chapelain. Deux facteurs conditionnent sa nomination: le prestige que procurent de riches prébendes et des qualités reconnues d'administrateur. Chaque année, il reçoit une somme globale afin de pourvoir à la rémunération, à l'entretien et aux frais divers. Selon la conjoncture, le nombre de chapelains varie de treize à dix-neuf, les clercs de chapelle de deux à six et les sommeliers de un à sept. En ce qui concerne leurs conditions de vie matérielle, les musiciens de la chapelle reçoivent, selon leur office, une pension quotidienne et annuelle, des valets pour leur service, des chevaux pour leurs fréquents déplacements et des subventions diverses pour leurs bons services, leurs études, l'acquisition de prébendes ou simplement subvenir à leurs besoins en cas de maladie. A ces gages, s'ajoutent les rémunérations qui se rattachent aux autres charges dont ils s'acquittent auprès du duc. Aussi, à l'exemple du personnel domestique de l'hôtel ducal, ils doivent se soumettre aux conventions vestimentaires qu'exige l'appartenance à la personne du prince. De même, des écussons à ses armes sont apposées sur leurs vêtements. D'ailleurs, à l'occasion des fêtes et des cérémonies religieuses et profanes, les musiciens revêtent des tenues et portent des couleurs que commandent les circonstances et la nature des sentiments.

Au plan pédagogique, les fondations de maîtrises d'enfants de choeur au sein des cathédrales et des chapelles princières permettent la constitution d'un bon réservoir de chantres et d'organistes. Sous l'autorité d'un maître recruté par le duc, les enfants recevront une éducation latine, religieuse et musicale et, dans ce dernier cas théorique et pratique, inspirée de Paris. Au chapitre de leur provenance, selon les éléments disponibles, les analyses onomastiques et toponymiques révèlent que sous le règne de Philippe le Hardi et de Jean Sans Peur, les compositeurs proviennent des régions du nord de la France, notamment de la région parisienne. Toutefois, sous Philippe le Bon et Charles le Téméraire, ils proviennent en majeure partie des régions artésiennes et flamandes. Peu de détails nous parviennent de leur milieu familial. Cependant, le type d'éducation reçu laisse supposer que les compositeurs émergent de familles aisées.

Mais fondamentalement, ces chapelains, compositeurs et fournisseurs 
de musique demeurent des hommes de leur siècle, hommes d'Eglise et hommes de cour. Hommes de leur siècle, ils partagent la vie et l'angoisse de leurs contemporains, vie teintée par la supersitition et par la libéralité des moeurs, angoisse que tente d'évacuer la fête des Fous qui, à l'exemple des danses macabres, masque la précarité de la condition humaine et la fragilite de toute position sociale. Hommes d'Eglise et surtout d'une Eglise complice de l'accroissement de la puissance bourguignonne, les chapelains pourvus de prébendes canoniales conférées par le duc, devront servir les intérêts de leur maitre au sein des chapitres et des assemblées auxquelles ils participent. Hommes de cour, les compositeurs se présentent comme des fournisseurs de musique de circonstance répondant à une demande sociale précise. Par conséquent, leur oeuvre, se développant dans une grande uniformité de style, n'offre rien de novateur. Elle demeure essentiellement profane, inspirée par les thèmes de l'idéal courtois. Secondaire, la musique sacrée ne propose aucune oeuvre véritablement profonde. D'ailleurs, ces compositeurs, même les plus célèbres, n'obtiennent jamais la haute dignité de premier chapelain et de conseiller ducal. Aussi, ceux-ci ne laisseront-ils aucune trace à titre de compositeurs.

\section{Les praticiens de la musique: les ménestrels de la cour}

A la fin du XIVe siècle, la jonglerie, industrie complexe florissant au cours des deux derniers siècles, se fragmente en une série de spécialités distinctes et isolées. La misère sévissant à l'aube du XVe siècle ne permet plus au jongleur de vivre de ses activités de divertissements auprès des strates moyennes et inférieures des milieux urbain et rural. Le seul marché potentiellement valable au plan économique reste celui des cours seigneuriales et princières. Aussi, y afflueront-ils, pour s'attirer la faveur des mécènes. S'il ne peut décrocher un poste au sein de ces domesticités, l'association, le regroupement confraternel et, plus tard, corporatif lui permettra d'espérer une situation plus sûre au plan financier et d'améliorer, en général, ses conditions de vie matérielle. Engagé suite à des auditions auprès du duc, le musicien reçoit alors le titre de "ménestrel." Du latin "ministerialis" signifiant subordonné, serviteur, domestique, le mot "ménestrel" ne possède donc d'abord aucune connotation avec le musicien. Le terme servait à propos des gens de maison, de bas officiers. Les ménestrels se classeront selon un ordre hiérarchique déterminé par les attributs moraux et sociaux des instruments sur lesquels ils s'exécutent et, par conséquent, selon les gens auxquels ils se subordonnent. Ainsi, à la cour bourguignonne, les ménestrels de hauts instruments, et parmi eux, les trompettes de guerre, restent-ils associés à leur maitre le duc, et les ménestrels de bas instruments, plus particulièrement les harpistes et les vielleurs, à la duchesse et au comte de Charolais. Le processus de fragmentation de l'exécution musicale qui sépare de plus en plus nettement l'élément poético- 
littéraire de l'exécution instrumentale tendra à accentuer chez le ménestrel sa simple fonction d'instrumentiste. Ces modifications se traduiront dans la terminologie: si le mot "ménestrel" reste encore de mise au XVe siècle, dès le début du siècle suivant, on ne parlera plus que des "ménétriers" et de plus en plus de "joueurs d'instruments."

Les états journaliers de la dépense de l'hôtel ducal rendent possible l'étude de quelques aspects de la vie quotidienne et domiciliaire des ducs et offrent de nombreuses précisions quant au personnel des divers offices. Cette domesticité nombreuse se compose d'un personnel stable, lié au duc par un rapport de subordination immédiat et quotidien. Dans un essai patronymique sur la domesticité de l'hôtel de Philippe le Bon, Henri David remarque une tendance toute naturelle à préciser l'individu par son rôle dans l'économie domestique. Ainsi, le type le plus courant et le plus répandu de ces dénominations se compose de deux termes: le premier baptismal, le second selon le rôle. "L'intéressé se précise donc tout ensemble sous l'angle chrétien et sous l'angle utilitaire."34 En premier lieu, les analyses onomastiques et toponymiques laissent clairement apparaitre le cosmopolitisme des musiciens au début du XVe siècle, dernier souffle du nomadisme des jongleurs médiévaux. De la même façon indiquent-elles le déclin de ce cosmopolitisme et, par conséquent, les traces de la sédentarisation puisque, de plus en plus, les ducs de Bourgogne, notamment Philippe le Bon et Charles le Téméraire, recruteront le personnel de leur maison au sein même de leurs territoires surtout ceux du Nord. ${ }^{35}$ De même, un projection des offices de l'hôtel se manifeste sur les dénominations de personnel. Par conséquent, l'énoncé du rôle de l'individu dans la familiarité domestique peut s'avérer un trait caractéristique de la subordination. Quelques instrumentistes prennent le nom de leurs instruments, l'objet précisant l'homme et gagnant, en cela, vertu d'emblême. ${ }^{36}$

L'appareil sonore des ducs se compose, en majeure partie, des ménestrels de hauts instruments. Leur fonction socio-musicale, et particulièrement celle des trompettes de guerre, consiste à traduire aux plans sonore et visuel la dignité, la magnificence et la force du prince. Ils ponctuent les manoeuvres militaires, proclament son entrée dans ses bonnes villes, annoncent les principales étapes de sa journée, publient les décrets et les arrêtés, s'exécutent dans les fanfares et rythment les diverses phases des joutes et tournois. Les sons qu'ils projettent exercent une fonction de signal. L'importance de la projection de l'autorité par des signaux sonores transforme ces hauts instrumentistes en agents de transmission d'informations ponctuelles exigeant une réponse précise et immédiate ou attirant l'attention sur les gestes du duc. Ils assument leur fonction à la guerre où l'on utilise le bruit d'abord à des fins stratégiques. L'attaque d'une ville ou d'une armée ennemie commence par le bruit délibérément éclatant voire violent des trompettes et des clairons menant la marche suivis par les 
autres hauts instrumentistes. Les chroniqueurs demeurent sensibles à l'intensité du bruit, de la "noise" provoquée par les hauts instruments. De la même manière, la prise de possession d'une ville se manifeste d'abord par l'entrée des trompettes du vainqueur dans la ville conquise. Plus spécifiquement, les trompettes de guerre remplissent une fonction psychologique, servant à la fois à raviver l'ardeur guerrière des troupes et à intimider l'ennemi. Outre les campagnes militaires, les hauts instrumentistes doivent accompagner le duc au cours de ses voyages officiels. Si l'escorte sonore varie selon l'importance des déplacements, restent toujours présents les trompettes de guerre et les autres ménestrels de hauts instruments. Chaque voyage offre l'occasion au duc de montrer sa magnificence et de déployer tout le faste que son pouvoir permet. Ainsi, sa puissance s'affirme, dans une certaine mesure, selon la quantité des instruments employés afin de dégager tout l'effet qu'il exige pour se faire annoncer. La présence des hauts instrumentistes s'avère particulièrement nécessaire quant à l'animation des fêtes, à la solennité des cérémonies, des joutes et des tournois. En ce qui concerne les cérémonies de la Toison d'or, ils doivent traduire ostensiblement au plan sonore à la fois l'ordonnance et la dignité de la cérémonie et des banquets. Selon l' "Espitre pour tenir et célébrer la noble feste du Thoison d'or" de l'historiographe Olivier de La Marche, les instrumentistes interviennent au moment du dîner, plus précisément pour la présentation des mets devant les convives. ${ }^{37}$ En ce qui a trait aux joutes et tournois, ces divertissements favoris de la noblesse exigent un déploiement sonore conforme à la mentalité et aux idéaux qui s'y projettent. Aussi restent-ils l'affaire presque exclusive des trompettes de guerre et des autres hauts instrumentistes. ${ }^{38}$ Les chroniqueurs mentionnent régulièrement dans leurs textes le grand bruit que suscitent ces exhibitions et insistent sur l'importance du cortège sonore glorifiant le héros de ces manifestations viriles et guerrières. Au chapitre des danses, l'importance du spectacle tend à s'effacer devant le plaisir social naissant de la participation à un divertissement où le spectateur auditeur devient acteur. L'exécution des danses exige une bonne diffusion des sons. Les musiciens prennent donc place sur un échaffaud aménagé à cet effet. ${ }^{39}$ Les circonstances semblent décider du choix des instruments utilisés: les caroles et les moresques demandent des hauts instruments alors que les basses danses requièrent des bas instruments.

Au chapitre de l'activité diplomatique, deux facteurs interviennent dans l'organisation d'une ambassade temporaire ordinaire au XVe siècle: la qualité des ambassadeurs et l'apparat matériel suivant le principe du nombre, et ce, dans le but d'attirer l'attention auditive et visuelle. La cour de Bourgogne éclipse toutes les autres par l'éclat et le luxe déployés lors des entrevues, alliant le plus large apparat à la stricte observance du droit des gens et de l'étiquette. Lors de l'entrée des ambassadeurs dans les villes, le cortège pénètre au bruit des canons et des trompettes. Au plan extra- 
musical, l'immunité nécessitant la production de pouvoirs réguliers est assurée aux ambassadeurs résidents ou temporaires, aux ambassadeurs d'apparat, aux hérauts et trompettes en mission et aux ambassades secrètes régulièrement accréditées. Souvent, des ambassadeurs envoyés en mission partent avec leurs homologues étrangers dans un but de sureté et de commodité. Ainsi, en temps de guerre, une bonne précaution, usuelle et facile pour traverser les lignes ennemies et faire reconnaître des envoyés, consiste à prendre un héraut ou un trompette comme escorte..$^{40}$ De même, les relations entre les cours s'apparentent à des échanges de bons procédés se maintenant souvent par l'entremise de leurs musiciens eux-mêmes. Les missions extra-diplomatiques se composent de certains agents jouant un rôle particulier: les rois d'armes, les hérauts, les poursuivants d'armes, les huissiers d'armes, les sergents d'armes et les trompettes de guerre. Ceuxci, par le prestige de leur fonction, jouissent, de tout temps et universellement, du droit absolu de circuler librement en tout pays à titre de parlementaire, et ce, même en temps de guerre, sur la simple justification d'un ordre émanant des autorités militaires de leur pays. A ce titre, ils amorcent des négociations entre les antagonistes. Les trompettes de guerre occupent plus fréquemment le rôle d'agents du duc lors de missions secrètes dont "il ne veult aultre declaracion estre faicte," de porteurs de dépêches, d'envoyers secrets voire d'espions militaires. ${ }^{41}$

Loin de susciter l'ardeur virile et guerrière, le rôle des ménestrels de bas instruments, les harpeurs, luthistes et vielleurs, reste tributaire de celui que la société du temps accorde à la musique. Par conséquent, ces bas instrumentistes ne se verront utilisés que pour agrémenter les banquets et les danses lors des fêtes, solennités, joutes et tournois. Mais avant toute chose, les chroniqueurs restent très imprécis dans l'identification de ces instrumentistes. L'utilisation de certains verbes, comme "sonner" ou "corner" pour les hauts instruments et "jouer" pour les bas, et de certaines expressions telles que "autres divers instrumens," laisse présumer des différenciations permettant de les identifier quelque peu.

D'abord, l'armée se servira du talent de ces ménestrels à des fins psychologiques, leur musique servant à divertir les chevaliers entre deux combats, et à des fins stratégiques, leurs chansons aux propos diffamatoires cherchant à intimider l'ennemi. De même, puisque les bas instrumentistes composent une partie des effectifs d'une ambassade temporaire, ils peuvent, en donnant des concerts, remplir leurs missions sans éveiller de méfiance. Ils se prêtent à des actions secrètes beaucoup plus hasardeuses et compromettantes que celles remplies par les trompettes de guerres et les autres hauts instrumentistes, et ce, même s'ils ne jouissent pas des mêmes immunités et avantages. Ils agissent à titre d'espions et peuvent, dans certains cas, se charger d'un attentat. ${ }^{42}$ Toujours sous le couvert d'exhiber leur talent, les bas instrumentistes agissent aussi à titre de porteurs de 
dépêches et de missives.

Les ménestrels de bas instruments, surtout les harpeurs, employés à titre de valets de chambre par le duc n'exerceront aucun travail proprement musical avant 1430, sauf pour l' "esbattement" et l'éducation musicale des jeunes enfants. D'ailleurs, s'appuyant sur l'iconographie. R. Wangermée souligne que pour les nobles "la musique était souvent associée à l'amour, tout en lui attribuant une grande importance dans leurs divertissements, peut-être la méprisaient-ils un peu." ${ }^{43}$ Donc, avant 1430, les harpeurs occupent le poste de valets de chambre du duc et de la duchesse. Sous Philippe le Hardi, obtenir cette charge domestique constitue pour les harpeurs un honneur auquel ils n'aspirent que très tard. Cependant, sous Philippe le Bon, Jeanne Marix remarque avec justesse que "Le titre de valet de chambre n'est pas pour Philippe le Bon un honneur qu'il accorde aux harpistes, mais la fonction de harpiste est plutôt une distraction qu'il tolère de ses valets de chambre." ${ }_{44}$ Aussi, ne semblent-ils engagés qu'à des fins purement domestiques pendant les premières années du règne de Philippe. Toutefois, après 1430, la présence à la cour d'Isabelle de Portugal s'avèrera bénéfique pour eux: désormais tout en exerçant leurs charges domestiques auprès du duc et de la duchesse, ils pourront faire valoir leur talent de musicien auprès de celle-ci.

Au chapitre des conditions de vie matérielle, des ménestrels de la cour ducale, les détails abondent en ce qui concerne les hauts instrumentistes. De loin le groupe le plus nombreux, au moins douze bon an mal an, ils bénéficient de pensions quotidiennes et annuelles. A ces gages réguliers s'additionnent, à l'occasion des fêtes religieuses et profanes, des voyages et des missions spéciales, des gratifications particulières. Les diverses solennités offertes à la cour ducale constituent de bonnes occasions pour les instrumentistes à l'affût de bénéfices supplémentaires de garnir leurs bourses. Propriétaires de biens meubles et immeubles, le duc leur accorde des gages supplémentaires afin qu'ils puissent à l'occasion retourner dans leurs familles. Les femmes de ménestrels participent, dans une certaine mesure, au statut de leurs époux en bénéficiant des largesses de la duchesse. Les ménestrels de hauts instruments doivent se conformer aux exigences vestimentaires de leur protecteur. D'abord, les trompettes de guerre arborent les couleurs ducales sur leurs vêtements et sur les bannières de leurs instruments. Ces vêtements adoptent le plus souvent la couleur vermeille, brodés aux armes du prince. Ces exigences vestimentaires prennent toute leur importance et leur signification lors de cironstances qui demandent un déploiement de couleurs appropriées. De même, ils reçoivent à leur entrée dans la domesticité ducale, un "émail" et un blason aux armes ducales.

Par contre, les ménestrels de bas instruments constituent le groupe le moins nombreux. Avant 1430, Philippe le Bon n'en possède qu'un seul de façon permanente, occupant le poste de valet de chambre. Après son 
mariage avec Isabelle de Portugal, le nombre de bas instrumentistes se fixera à quatre. A l'exemple de leurs confrères, ils bénéficient de pensions quotidiennes et annuelles, de diverses gratifications et rentes. Cependant, les comptes de Philippe le Bon et de Charles le Téméraire demeurent très imprécis sur leurs costumes. Toutefois, selon l'état de la domesticité du dernier grand duc de Bourgogne, ils doivent porter ses armes, à l'exemple des gens de sa maison.

A l'amorce du processus de sédentarisation des musiciens dans les cours, à la fin du XIVe et au début du XVe siècle, le principal critère de sélection réside dans l'habileté exceptionnelle de l'instrumentiste, engagé après une audition auprès du duc lui-même. Après la formation d'un noyau de bons ménestrels, le duc s'attachera de très jeunes gens auxquels ils confiera l'éducation à des musiciens éprouvés, s'inspirant en cela des méthodes d'apprentissage de l'organisation des métiers. L'apprenti reçoit le titre de petit ménestrel et reste parfois de langues années à suivre les enseignements de son aîné. De la même manière, celui-ci transmettra son art et son métier à sa progéniture. A l'encontre des ménestrels de hauts instruments, les bas instrumentistes participent aux écoles de ménestrels, réunions périodiques se tenant dans une ville fixée à l'avance au temps du carême, quand la musique et les autres manifestations de divertissement demeurent interdites. Il s'agit de congrès où les ménestrels de différents pays, obtenant des sommes d'argent de leurs protecteurs, se rencontrent afin d'échanger, écouter et apprendre de nouvelles chansons leur permettant d'étendre leur répertoire. J. Marix constate que les mentions d'écoles s'avèrent nombreuses à la fin du XIVe siècle sous Philippe le Hardi. ${ }^{45}$ Cependant, sous Philippe le Bon, aucun compte n'indique l'envoi de ménestrels ducaux à ces écoles. D'abord, Philippe n'attache guère d'importance à l'engagement de ménestrels de bas instruments, préférant gratifier les ménestrels de passage ou ceux des villes lors de ses voyages. Plus nombreux depuis l'arrivée de la duchesse Isabelle, ils semblent évoluer en vase clos, encadrés par la cour et renouvellant leur répertoire lors de la venue de musiciens itinérants qu'ils doivent alors écouter. Toutefois, des conditions d'ordre artistique imposeront, dans la deuxième moitié du XVe siècle, aux ménestrels de profonds changements d'organisation et d'esthétique résidant au sein d'une plus large parcellisation ou fragmentation de l'exécution musicale. Dans un premier temps, la musique vocale se passera d'accompagnement instrumental. D'autre part, les instrumentistes se confineront au jeu de leurs instruments et se serviront de plus en plus des pratiques d'apprentissage de l'organisation des métiers. Le ménestrel, musicien et poète du siècle précédent, devient un ménétrier, un simple exécutant qui se préoccupe de moins en moins de l'élément littéraire pour se spécialiser dans la musique instrumentale. Ainsi, la spécialisation, des instrumentistes, la sédentarisation, le mode d'apprentissage et le renouvellement du 
répertoire dans les lieux mêmes d'où émane la demande sociale rendent désuettes les "escolles" de ménestrels dès la deuxième moitié du XVe siècle. Du mème souffle, disparaissent l'éclectisme et le nomadisme des premiers temps au profit du recrutement des jeunes dans les possessions ducale et de l'héridité qui tendront à accentuer le caractère flamand du groupe des musiciens de la cour de Bourgogne dont le centre semble se fixer dans les villes du Nord au cours du siècle.

Ainsi, plus que de simples protecteurs, les Grands Ducs de Bourgogne se présenteront plutôt comme des employeurs auxquels se subordonnent des musiciens salariés, la musique étant beaucoup plus, à la fin du Moyen Age, un métier aux applications pratiques qu'un art aux prétentions esthétiques:

\begin{abstract}
... la musique ètait beaucoup plus un métier qu'un art. Si elle commençait à être reconnue comme un art, ce n'était pas un art pur que l'on pouvait soustraire de sa réalité sociale; ce n'était qu'un art fonctionnel, le décor sonore de la vie quotidienne. C'est parce qu'elle était liee à la vie, aux goûts, aux sentiments, à la mode, que la musique s'intégrait si rigoureusement dans le présent. Religieuse et profane, visant à l'édification ou au divertissement, elle se transformait sans cesse pour répondre à l'évolution spirituelle et culturelle du groupe qu'elle voulait satisfaire. ... ${ }^{46}$
\end{abstract}

Par conséquent, un type de causalité circulaire s'établit: le musicien se classera dans la société selon l'échelle des valeurs prédominant dans le milieu de son activité, selon la hiérarchie des instruments de musique conçue d'après un vaste appareil symbolique, ainsi que selon la fonction et la destination sociale pratique du produit musical. Les attitudes collectives des musiciens envers eux-mêmes changent en fonction des groupes auxquels ils appartiennent et se subordonnent, de même qu'en fonction des rôles sociaux qu'ils assument. Aussi, les diverses dénominations du musicien à la fin de l'époque médiévale reflètent une différence de conception de l'importance voire de la dignité de sa personne, de l'inégalité de sa condition selon le milieu social de son activité.

\title{
Université de Montréal
}

\section{Notes}

1 Jacques Attali, Bruits (Paris: P.U.F., 1977), p. 93.

2 Bernard Guenée, L'Occident aux XIVe et XVe siècles. Les Etats (Coll. Nouvelle Clio, 22; Paris: P.U.F., 1971), p. 85.

3 A cet effet, nous disposons des recueils de MM. Louis P. Gachard, Ernest Petit et Herman Vander Linden qui dressent, à l'aide d'extraits de la comptabilité ducale, les itinéraires des quatre ducs Valois de Bourgogne. Sur la mème base de recherche, nous disposons des pièces comptables rassemblées par le comte Léon de Laborde pour son étude sur les lettres, les arts et l'industrie au XVe siècle ainsi que des travaux de Mme Jeanne Marix sur l'histoire de la musique et des musiciens au temps de Philippe le Bon, travaux basés sur le dépouillement d'un grand nombre de comptes tirés de la recette générale des finances des ducs. 


\section{4 / Renaissance and Reformation}

4 Il s'agit des chroniques bourguignonnes du XVe siècle notamment celles de Jean Lefèvre, seigneur de Saint-Remy, d'Enguerrand de Monstrelet, de l'historiographe Georges Chastellain, de Mathieu d'Escouchy, du mémorialiste Jacques Du Clercq, d'Olivier de La Marche et de Jean Molinet. De même, nous recourrons aux relations dues à des particuliers curieux de conserver le souvenir des événements importants: Dom Antoine de la Taverne et Jean de Roye. Enfin, nous utiliserons quelques extraits de certains textes littéraires surtout ceux d'Antoine de La Sale, de Martin le Franc, de Nicole de la Chesnaye et d'Eloi d'Amerval.

5 L'école franco-flamande de la fin du XIVe et du XVe siècle manifeste une tendance au réalisme que traduit l'abondance de musique dans les peintures. Même si parfois les exigences du langage plastique les éloignent délibérément d'une représentation littérale et rigoureuse, l'oeuvre des artistes flamands constitut la seule source concrète et éloquente de l'histoire des effectifs sonores de l'èpoque.

6 Theodor W. Adorno, "Sociologie de la musique," dans Musique en jeu, 2(1971), 7-15; "Reflexions en vue d'une sociologie de la musique," dans Musique en jeu, 7 (1972), 5-15; Ivo Supicic, Musique et société (Zagreb: Institut de musicologie, 1971), 205.

7 André Schaeffner, "Musique populaire et art musical," dans Journal de psychologie, nos 1-2 (1951), p. 253.

8 Françoise Escal, Espaces sociaux espaces musicaux (Paris: Payot, 1979), p. 38.

9 J. Attali, p. 37.

10 Joseph Calmette, Les Grands Ducs de Bourgogne (Paris: Albin-Michel, 1949), p. 298.

11 Jacques Le Goff, "Au Moyen Age: temps de l'Eglise et temps du marchand," dans Annales E.S.C., 15 (1960), 417-33; "Le temps du travail dans la "crise" du XIVe siècle: du temps médiéval au temps moderne," dans Le Moyen Age, 69 (1963), 597-613.

12 Johan Huizinga, Le déclin du Moyen Age (trad. du hollandais par J. Bastin; nouv. éd., coll. Petite Bibliothèque Payot, 108; Paris: Payot, 1967), pp. 10-1.

13 Georges Chastellain, “Chronique,” dans Oeuvres (Nouv. èd.; Genève; Slatkine Reprints, 1971), $\mathrm{V}, 364$.

14 J. Calmette, p. 179.

15 J. Huizinga, pp. 284-5.

16 Mentionnons le cas bien connu de Charles d'Orléans qui, à 19 ans, se fait confectionner une robe aux manches brodees du "dit" de la chanson Madame je suis plus joyeulx et dont 568 perles dessinent les notes de la mélodie. Cf. Pierre Champion, Vie de Charles d'Orléans(1394-1465) (Paris, 1911), p. 132.

17 Edmond A. Bowles, "Instruments at the Court of Burgundy (1363-1467)," dans Galpin Society Journal, 6 (1953), 41-51; "Haut and Bas: the Grouping of Musical Instruments in the Middle Ages," dans Musica Disciplina, 8 (1954), 115-40.

18 Raymond Murray Schafer, Le paysage sonore (Coll. Musiques et Musiciens; J.-C. Lattès, 1979), p. 246.

19 J. Huizinga, p. 107.

20 F. Escal, p. 219.

21 B. Guenée, op. cit.

22 Jeanne Marix, Histoire de la musique et des musiciens de la Cour de Bourgogne sous le règne de Philippe le Bon (1420-1467) (Strasbourg: Heitz \& Co., 1939), p. 1.

23 Antoine Goléa, La musique dans la société européenne depuis le Moyen Age jusqu'à nos jours (Paris: Témoignage chrétien, 1960), p. 7.

24 J. Le Goff, "Métiers licites et métiers illicites dans l'Occident médiéval," dans Pour un autre Moyen Age (Paris: Gallimard NRF, 1977).

25 Guillaume de Van, "La pédagogie musicale à la fin du Moyen Age," dans Musica Disciplina, II, 1-2 (1948), 78.

26 Une miniature de 1441, extraite du Champion des Dames de Martin le Franc, représente Maistre Guille du Fay et Binchois. Le premier porte une robe bleue d'allure cléricale avec, à ces cotés, un petit orgue portatif et le deuxième une tunique rouge nouée à la taille par une ceinture de cuir à bou- 
cle de métal et sa main gauche tient une harpe. Ce document iconographique demontre l'importance de Dufay dans la musique sacree et celle de Binchois dans la musique profane. Cf. Charles Van den Borren, "Guillaume Dufay: son importance dans l'èvolution de la musique au XVe siècle," dans Bulletin de l'Académie royale de Belgique (Bruxelles, 1926), pp. 34-5.

27 Olivier de La Marche, "S"ensuyt l'estat de la maison du duc Charles de Bourgoingne, dit le Hardy,"'dans Memoires (H. Beaune-J. d'Arbaumont, ed.; Paris: Renouard, 1887), IV, 2.

28 J. Marix, pp. 80-1.

29 Ibid., p. 175.

30 Ibid., p. 34.

31 Ernest Petit, Itinéraires de Philippe le Hardi et de Jean Sans Peur, ducs de Bourgogne (13631419), d'après les comptes de dépenses de leur hötel, Coll. de documents inédits sur l'histoire de France, (Paris: Imprimerie nationale, 1888), XLIX, 614.

32 M. du Tilliot, Memoires pour servir à la féte des Fous, p. 102, dans J. Marix, op. cit., p. 53.

33 Edouard de Moreau, Histoire de l'Eglise en Belgique (Bruxelles: l'Edition universelle, 1949), IV, 72. L'auteur souligne le cas pertinent du chapitre de Sainte-Waudru de Mons. De 1427 à 1483, 65 nominations de chanoines et de chanoinesses procèdent de Philippe le Bon, Charles le Téméraire, Maximilien d'Autriche et de Marie de Bourgogne. Sur 25 personnages promus à la dignité canoniale, sept. seulement n'exerceront aucune fonction à la cour du prince.

34 Henri David, "Les offices de l'hôtel ducal sous Philippe le Bon. Essai patronymique," dans Annales de Bourgogne, 35 (1963), 211.

35 J. Marix, op. cit., p. 122.

36 H. David, "Les officiers ducaux sous Philipppe le Bon," dans Annales de Bourgogne, no 37 (Dijon, 1965), 16.

37 O. de La Marche, "Espitre pour tenir et célébrer la noble feste du Thoison d'or," dans Mémoires (H. Beaune-J. d'Arbaumont, éd.: Paris: Renouard, 1887), IV, 182.

38 En 1462, un valet de chambre de Philippe le Bon, Jean Dreux, exécute une miniature représentant une scène de tournoi oủ participent deux trompettes et un joueur de saqueboute. Cf. L.-M.-J. Delaissé, La Miniature flamande. Le mécénat de Philippe le Bon, catalogue d'exposition (Bruxelles, avril-juin 1959).

39 Jean Lefevre de Saint-Remy, Chronique (Francis Morand, ed.; Paris: Renouard, 1881), II, $160-1$.

40 Rene Alphonse de Maulde-de-la-Clavière, La diplomatie au temps de Machiavel (Nouv. èd.; Genève, Slatkine Reprints, 1970), II, 63-4.

41 J. Marix, pp. 57-8, note 5.

42 Andre Pirro, La musique à Paris sous le règne de Charles VI(1380-1422) (Strasbourg: Heitz \& Co., 1930), pp. 9-10.

43 Robert Wangermée, La musique flamande dans les sociétés des XVe et XVIe siècle (Bruxelles: Arcade, c1965), p. 208.

44 J. Marix, p. 89, note 2.

45 Ibid., p. 97 , note 3.

46 R. Wangermèe, p. 68. 